\title{
ON THE ISOMETRIC IMMERSIONS IN EUCLIDEAN SPACE OF MANIFOLDS WITH NONNEGATIVE SECTIONAL CURVATURES. II $\left({ }^{1}\right)$
}

\author{
BY \\ PHILIP HARTMAN
}

1. The main theorems. The object of this note is to prove the following:

THEOREM 1.1. Assume that (i) $M^{d}$ is a d-dimensional ( $\left.d \geqq 2\right)$, complete Riemann manifold of class $C^{k}, k \geqq 2$, with nonnegative sectional curvatures; (ii) $f: M^{d} \rightarrow E^{d+e}$ is a $C^{k}$ isometric immersion of $M^{d}$ into a Euclidean space $E^{d+e}, e>0$; and (iii) the relative nullity $\nu(p)$ of the immersion $f$ at the point $p \in M^{d}$ satisfies $\nu(p)>0$ for all $p$. Let $m=\min \nu(p)$ for $p \in M^{d}$. Then $f\left(M^{d}\right)$ is $C^{k-1} m$-cylindrical; that is, there exists a complete Riemann manifold $M^{d-m}$ of class $C^{k-1}$ such that $M^{d}, E^{d+e}, f$ have factorizations $M^{d}=E^{m} \times M^{d-m}, E^{d+e}=E^{m} \times E^{d+e-m}, f=i \times g$, where $i: E^{m} \rightarrow E^{m}$ is the identity map and $g: M^{d-m} \rightarrow E^{d+e-m}$ is a $C^{k-1}$ isometric immersion. Also, $f\left(M^{d}\right)$ is not $(m+1)$-cylindrical.

A vector in $T_{p}\left(M^{d}\right)$, the tangent space of $M^{d}$ at $p \in M^{d}$, is called a relative nullity vector (with respect to $f$ ) if it is annihilated by all second fundamental matrices at $p$. The relative nullity $\nu(p)$ is the dimension of the linear space of relative nullity vectors in $T_{p}\left(M^{d}\right)$; Chern and Kuiper [3].

The conclusion of Theorem 1.1 is stated with a slight abuse of language which will be followed consistently below. The statement " $M^{d}=E^{m} \times M^{d-m}$ " means that "there exists an isometric diffeomorphism $I$ of $E^{m} \times M^{d-m}$ onto $M^{d}$ (so that $I \in C^{k-1} ;[2]$ )" and correspondingly " $f=i \times g$ " should be replaced by " $f \circ I=i \times g$ ".

An example of Sacksteder [12, p. 623], shows that Theorem 1.1 is false if "nonnegative" is replaced by "nonpositive" sectional curvatures.

Theorem 1.1 was proved in Hartman [6] under the additional assumption $\nu(p) \equiv m>0$ for all $p \in M^{d}$; cf. O'Neill [10] and Stiel [13] for corresponding results under additional assumptions on the curvatures of $M^{d}$ and $f\left(M^{d}\right)$. For the case of hypersurfaces $(e=1)$, Theorem 1.1 is contained in a result of Sacksteder [12]. In the case that $M^{d}$ is locally flat and $d>e$, Theorem 1.1 has the following consequence.

Corollary 1.1. Assume that (a) $M^{d}$ is a d-dimensional ( $\left.d \geqq 2\right)$, complete Riemann manifold of class $C^{k}, k \geqq 2$, with vanishing sectional curvatures; (b)

Received by the editors August 19, 1969.

( $\left.{ }^{1}\right)$ Research partially sponsored by the Air Force Office of Scientific Research, Office of Aerospace Research, United States Air Force, under AFOSR Contract No. F44620-67-C-0098, and by the Science Research Council of Great Britain.

Copyright (C) 1970, American Mathematical Society 
$f: M^{d} \rightarrow E^{d+e}$ is a $C^{k}$ isometric immersion; and (c) $d>e$. Then $m=\min v(p)$ is positive and the conclusion of Theorem 1.1 holds with $M^{d-m}, g \in C^{k}$.

In fact, (a) and (b) imply that $\nu(p) \geqq d-e$; [3]. If $e=1$ (so that $f\left(M^{d}\right)$ is a hypersurface in $\left.E^{d+1}\right)$, then $\nu(p) \geqq d-1>0$ and Corollary 1.1 reduces to a theorem of Hartman and Nirenberg [8].

On a Riemann manifold $M^{d}$ with a continuous curvature tensor, a vector $z \in T_{p}\left(M^{d}\right)$ is called a nullity vector at $p$ if $R(x, y) z=0$ for all $x, y \in T_{p}\left(M^{d}\right)$, where $R(x, y)$ is the curvature transformation associated with $x, y$; the nullity $\mu(p)$ is the dimension of the linear space of nullity vectors in $T_{p}\left(M^{d}\right)$; Chern and Kuiper [3]. On a complete Riemann manifold $M^{d}$, Toponogov [15] defines a "line" to be a complete geodesic with every subarc of minimal length. Corollary 1.1 has the following generalization.

Corollary 1.2. Assume (i), (ii) of Theorem 1.1 and that the nullity satisfies $\mu(p)>e$ for all $p \in M^{d}$ (for example, that there exists $\mu_{0}$ linearly independent "lines" through some point $p_{0} \in M^{d}$ and $\left.\mu_{0}>e\right)$. Then $m=\min \nu(p)$ is positive and the conclusion of Theorem 1.1 holds.

Corollary 1.1 is the special case where $\mu(p)=d$ for all $p$ (by virtue of the local flatness). In general, Chern and Kuiper [3] prove that $\mu(p) \geqq \nu(p) \geqq \mu(p)-e$. As to the parenthetical part of Corollary 1.2, results of Toponogov [15] imply that $\mu(p) \geqq \mu_{0}$; cf. Proposition 4.2 below.

It is easy to see (cf. Proposition 4.1 below) that if assumptions (i), (ii) of Theorem 1.1 hold and $L^{1}$ is a geodesic arc on $M^{d}$ such that $L_{1}=f\left(L^{1}\right)$ is a line segment in $f\left(M^{d}\right) \subset E^{d+e}$, then a vector on $T_{p}\left(L^{1}\right)$ at $p \in L^{1}$ is a relative nullity vector at $p$ and the normal space of $f\left(M^{d}\right) \subset E^{d+e}$ is constant (i.e., parallel) along $L_{1}$. This implies that $f\left(M^{d}\right)$ cannot be $(m+1)$-cylindrical.

In Theorem 1.1, let $p_{0}$ be a point of $M^{d}$ where $\nu\left(p_{0}\right)=m$. Then $\nu(p)=\nu\left(p_{0}\right)$ for all $p$ near $p_{0}$ and, hence, by Lemma 3.1 of Hartman [6, p. 98], there exists for such $p$ a Euclidean space $E^{m}(p)$ satisfying $f(p) \in E^{m}(p) \subset f\left(M^{d}\right)$ (and, in fact, the spaces $E^{m}(p)$ are parallel; [6, pp. 100-101]). Thus, Theorem 1.1 is contained in the following

TheOrem 1.2. Assume (i), (ii) of Theorem 1.1 and that $f\left(M^{d}\right)$ contains $m$ linearly independent lines, $0<m \leqq d$. Then the conclusion of Theorem 1.1 holds.

The assumption that $f\left(M^{d}\right)$ contains $m$ "linearly independent" lines $L_{1}, \ldots, L_{m}$ means that translates of $L_{1}, \ldots, L_{m}$ through the origin of $E^{d+e}$ span an $m$-space.

In any of the statements above, there is no loss of generality in supposing that $M^{d}$ is simply connected, for otherwise it can be replaced by its universal covering manifold. If, in addition, the sectional curvatures of $M^{d}$ vanish, then it can also be supposed that $M^{d}=E^{d}$ cf., e.g., [8, p. 913]. Thus the reduction above of Theorem 1.1 to Theorem 1.2 shows that the flat cases of these theorems are contained in 
Lemma 1.1. Let $f: E^{d} \rightarrow E^{d+e}$ be an isometric immersion of class $C^{k}, k \geqq 1$, such that $f\left(E^{d}\right)$ contains $m$ linearly independent lines. Then there exist factorizations $E^{d}=E^{m} \times E^{d-m}, E^{d+e}=E^{m} \times E^{d+e-m}$, and $f=i \times g$ such that $i: E^{m} \rightarrow E^{m}$ is the identity map and $g: E^{d-m} \rightarrow E^{d+e-m}$ is a $C^{k}$ isometric immersion.

In contrast to the case $e=1$, where necessarily $m \geqq d-1$, the situation $m=0$ can occur in Lemma 1.1 when $e>1$. For example, if $u=\left(u^{1}, \ldots, u^{d}\right) \in E^{d}$ and $e=d$, one can choose the isometric immersion $f: E^{d} \rightarrow E^{2 d}$ to be of the form

$$
f\left(u^{1}, \ldots, u^{d}\right)=\left(f_{1}\left(u^{1}\right), g_{1}\left(u^{1}\right), f_{2}\left(u^{2}\right), \ldots, g_{d}\left(u^{d}\right)\right),
$$

where $\left(f_{k}, g_{k}\right): E^{1} \rightarrow E^{2}$ is an isometric immersion for $k=1, \ldots, d$. It is easy to see that if $f_{k}, g_{k}$ are suitably chosen, then $f\left(E^{d}\right) \subset E^{2 d}$ contains no lines. Actually, $m>0$ must hold only if $d>e$.

Below, we shall first prove Lemma 1.1 in $\$ 2$. (In fact, it will essentially be sufficient to consider only the case $d=2$ and $m=1$.) Theorem 1.2 will then be deduced in $\$ 3$ from Lemma 1.1 and a factorization theorem of Toponogov [15] which is the "intrinsic" analogue of Theorem 1.2. This gives a rather short proof of Theorem 1.1 and of the corresponding part of Sacksteder's result. Actually, the brevity is somewhat misleading for, in contrast to Sacksteder's proof, we employ Toponogov's theorem.

The arguments in the proof of Theorem 1.2 also give the following theorem (in which there is no assumption on the relation between curvatures of $M^{d}$ and $M^{d+e}$ ):

THEOREM 1.3. Let $\left(i_{0}\right) M^{d+e} \in C^{k+1}$ be a complete Riemann manifold with nonnegative sectional curvatures $(d \geqq 2, e>0, k \geqq 2)$; (i) $M^{d} \in C^{k}$ a complete Riemann manifold with nonnegative sectional curvatures; (ii) $f: M^{d} \rightarrow M^{d+e}$ an isometric immersion of class $C^{k}$ such that $f\left(M^{d}\right)$ contains $m(>0)$ linearly independent "lines" of $M^{d+e}$. Then there exist factorizations $M^{d+e}=E^{m} \times M^{d+e-m}, M^{d}=E^{m} \times M^{d-m}$, and $f=i \times g$ such that $M^{d+e-m} \in C^{k-1}, M^{d-m} \in C^{k-1}$ are complete Riemann manifolds, $i: E^{m} \rightarrow E^{m}$ is the identity map, and $g: M^{d-m} \rightarrow M^{d+e-m}$ is a $C^{k-1}$ isometric immersion.

The reader not interested in the question of differentiability conditions should read the assertions and proofs with all differentiability classes replaced by $C^{\infty}$. For other readers, $\S 5$ will contain remarks and clarifications of the differentiability classes which occur in the statements and proofs. For locally flat manifolds, there is no difficulty. In general, what is involved is a determination of conditions sufficient for the validity of Rauch's comparison theorem [11] and of Toponogov's comparison theorem [14] and factorization theorem [15].

2. Proof of Lemma 1.1. Case $1(d=2$ and $m=1)$. Let $L$ be a line in $f\left(E^{2}\right) \subset E^{2+e}$. Since $f$ is an isometry there exists a line $L_{1}$ in $f^{-1}(L) \subset E^{2}$ which $f$ maps homeomorphically onto $L$. Choose coordinates $(u, v)=\left(u^{1}, \ldots, u^{1+e} ; v\right) \in E^{2+e}$ such that $L: u=0$, where we write $u=\left(u^{1}, \ldots, u^{1+e}\right)$ and $v$ is real-valued. Choose coordinates 
$(r, s)$ in $E^{2}$ such that $L_{1}: r=0$ and that if $f(r, s)=(u(r, s), v(r, s))$, then

$$
u(0, s)=0 \text { and } v(0, s)=s .
$$

In order to complete the proof in Case 1, it suffices to show that $u(r, s)=u(r)$ is a function of $r$ alone and that $v(r, s)=s$,

$$
f(r, s)=(u(r), s) \text { for }-\infty<r, s<\infty .
$$

Below $|f|,|u|,|v|, \ldots$ denote Euclidean norms and a dot means scalar multiplication. Thus if $f_{r}=\partial f / \partial r$ and $f_{s}=\partial f / \partial s$, then

$$
\left|f_{r}\right|=\left|f_{s}\right|=1 \text { and } f_{r} \cdot f_{s}=0 .
$$

We have, by (2.3) that $|f(r, s)-f(0, s)| \leqq|r|$, i.e.,

$$
|u(r, s)|^{2}+|s-v(r, s)|^{2} \leqq r^{2} .
$$

In particular,

$$
\left|\int_{0}^{s}\left[1-v_{s}(r, t)\right] d t\right| \leqq|v(r, 0)|+|r|
$$

Since the inequality

$$
1-v_{s}(r, s) \geqq 0
$$

is clear from (2.3),

$$
\int_{-\infty}^{+\infty}\left|1-v_{s}(r, s)\right| d s \leqq|v(r, 0)|+|r|<\infty .
$$

In particular,

$$
v_{ \pm}(r)=\lim _{s \rightarrow \pm \infty}\left[s-v_{s}(r, s)\right] \text { exist. }
$$

From $\left|u_{s}\right|^{2}=\left|f_{s}\right|^{2}-\left|v_{s}\right|^{2}=\left(1+v_{s}\right)\left(1-v_{s}\right) \leqq 2\left(1-v_{s}\right)$ and from (2.6),

$$
\int_{-\infty}^{+\infty}\left|u_{s}(r, s)\right|^{2} d s \leqq 2(|v(r, 0)|+|r|)<\infty .
$$

By (2.3), $f_{r} \cdot f_{s}=u_{r} \cdot u_{s}+v_{r} v_{s}=0$ or, equivalently, $v_{r}=-u_{r} \cdot u_{s}+\left(1-v_{s}\right) v_{r}$. Hence $\left|v_{r}\right|^{2} \leqq 2\left(\left|u_{s}\right|^{2}+\left|1-v_{s}\right|^{2}\right) \leqq 2\left|u_{s}\right|^{2}+4\left|1-v_{s}\right|$, so that

$$
\int_{-\infty}^{+\infty}\left|v_{r}(r, s)\right|^{2} d s \leqq 8(|v(r, 0)|+|r|)<\infty .
$$

Consequently, by Schwartz's inequality,

$$
\int_{-\infty}^{+\infty}\left|\int_{0}^{r} v_{r}(t, s) d t\right|^{2} d s \leqq|r| \int_{-\infty}^{+\infty} \int_{0}^{r}\left|v_{r}(t, s)\right|^{2} d t d s<\infty
$$

Hence $v(0, s)=s$ gives

$$
\int_{-\infty}^{+\infty}|s-v(r, s)|^{2} d s<\infty
$$


It follows from (2.7) that $v_{ \pm}(r) \equiv 0$. But, for fixed $r, s-v(r, s)$ is a nondecreasing function of $s$ by $(2.5)$, so that $v(r, s) \equiv s$.

The relation $1=\left|f_{s}\right|^{2}=\left|u_{s}\right|^{2}+\left|v_{s}\right|^{2}=\left|u_{s}\right|^{2}+1$ shows that $u_{s}(r, s) \equiv 0$, i.e., $u(r, s)$ $=u(r)$ is a function of $r$ alone. This proves (2.2) and Case 1 of Lemma 1.1.

Case $2(d \geqq 2$ and $0<m \leqq d)$. The proof for the Case 1 shows that if $L$ is a line in $f\left(E^{d}\right) \subset E^{d+e}$, then through every point of $f\left(E^{d}\right)$ there passes a line in $f\left(E^{d}\right)$ parallel to $L$. In particular, there are factorizations $E^{d}=D^{m} \times E^{d-m}$ such that $f\left(E^{m} \times\{0\}\right)$ contains $m$ linearly independent lines through the point $f(0,0)$ of $E^{d+e}$. Since $f$ is an isometry, it follows that $f \mid\left(E^{m} \times\{0\}\right)$ is a linear (isometric) transformation of $E^{m} \times\{0\}$ into an $m$-dimensional subspace of $E^{d+e}$. The proof of Case 1 now implies the general case.

3. Proof of Theorem 1.2. Following Toponogov [15], we call a complete geodesic in $M^{d}$ a "line" if every subarc is minimizing. He shows that if $L$ is a "line" in $M^{d}$, then, under the condition of Theorem 1.2 on $M^{d}$, through every point $p \in M^{d}$, there is a unique "line" $L(p)$ "parallel" to $L(i)$; cf. $\S 5$.

If two "lines" $L^{1}, L^{2}$ in $M^{d}$ are "parallel" in the sense of Toponogov, they possess arclength parametrization $g_{1}, g_{2}: E^{1} \rightarrow M^{d}$ and there exists a homotopy $g: E^{1} \times[0, T] \rightarrow M^{d}$ such that $g\left|\left(E^{1} \times\{0\}\right)=g_{1} ; g\right|\left(E^{1} \times\{T\}\right)=g_{2} ; g(s, \cdot):[0, T]$ $\rightarrow M^{d}$ is an arclength parametrization of a minimizing geodesic orthogonal to $L^{1}, L^{2}$ at $t=0, T$, respectively; and $g(\cdot, t): E^{1} \rightarrow M^{d}$ is an arclength parametrization of the unique "line" in $M^{d}$ parallel to $L^{1}$ and $L^{2}$ through the point $g(s, t)$. When $M^{d}$ is of class $C^{k}, k \geqq 2$, with a curvature tensor of class $C^{k-2}$, then $g \in C^{k-1}$; cf. $\S 5$.

Suppose that $L_{1}$ is a line of $E^{d+e}$ in $f\left(M^{d}\right)$. Then there exists a "line" $L^{1}$ in $f^{-1}\left(L_{1}\right)$ which $f$ maps homeomorphically onto $L^{1}$. The proof of Lemma 1.1 shows that if $\left\{L^{1}(p)\right\}, p \in M^{d}$, is the family of "lines parallel to $L^{1}$ ", then $L_{1}(p)=f\left(L^{1}(p)\right)$, $p \in M^{d}$, is a family of parallel lines in $E^{d+e}$.

If $L_{1}, \ldots, L_{m}$ are linearly independent lines of $E^{d+e}$ in $f\left(M^{d}\right) ; L^{1}(p), \ldots, L^{m}(p)$ corresponding families of "parallel lines" in $M^{d}$; and $L_{1}(p)=f\left(L^{1}(p)\right), \ldots, L_{m}(p)$ $=f\left(L^{m}(p)\right)$ the related families of parallel lines in $E^{d+e}$, then $L_{1}(p), \ldots, L_{m}(p)$ span an $m$-space at $f(p)$.

According to the factorization theorem of Toponogov [15] (cf. $\left(T_{\infty}\right)$ in $\left.\S 5\right)$, there is a factorization of $M^{d}$ of the form $M^{d}=E^{m} \times M^{d-m}$, where $M^{d-m} \in C^{k-1}$ is a complete Riemann manifold. If $p=\left(p_{0}, p_{1}\right) \in M^{d}$, with $p_{0} \in E^{m}$ and $p_{1} \in M^{d-m}$, then $L^{1}(p), \ldots, L^{m}(p)$ span $\left(E^{m} \times\left\{p_{1}\right\}\right) \subset M^{d}$. The map $f \mid\left(E^{m} \times\left\{p_{1}\right\}\right)$ is a linear isometric map of $E^{m} \times\left\{p_{1}\right\}$ onto an $m$-dimensional subspace of $E^{d+e}$ and, $f \mid\left(\left\{p_{0}\right\} \times M^{d-m}\right)=g\left(p_{1}\right)$ is independent of $p_{0}$; cf. the proof in Case 1 of Lemma 1.1 that $u_{s}=0$. This proves Theorem 1.2.

4. Nullity. The purpose of this section is to give two propositions which provide verifications for statements of $\S 1$ concerning nullity and relative nullity. 
Proposition. 4.1. Assume that $M^{d}$ is a d-dimensional $(d \geqq 2)$ Riemann manifold of class $C^{2}, f: M^{d} \rightarrow E^{d+e}$ a $C^{2}$ isometric immersion, and $L^{1} \subset M^{d}$ an arc of class $C^{1}$. Then the following are equivalent: (1) the tangent vectors to $L^{1}$ are relative nullity vectors and (2) the normal spaces of $f\left(M^{d}\right)$ are constant (i.e., parallel) along $L_{1}=f\left(L^{1}\right)$. If $M^{d}$ has nonnegative sectional curvatures and $L^{1}$ is a geodesic, then (1) and (2) are equivalent to: (3) $L_{1}=f\left(L^{1}\right) \subset f\left(M^{d}\right) \subset E^{d+e}$ is a line segment.

Proof. Fix a point $p_{0} \in L^{1}$ and let $u=\left(u^{1}, \ldots, u^{d}\right)$ be local coordinates in a neighborhood of $p_{0}$ on $M^{d}$ and let $f=f(u)=f\left(u^{1}, \ldots, u^{d}\right)$ give the embedding of this neighborhood. We shall use the following indices on the indicated ranges

$$
1 \leqq i, j, k \leqq d \text { and } 1 \leqq \kappa, \lambda \leqq e,
$$

and observe the usual tensor summation convention. Let $N^{\lambda}(u), \lambda=1, \ldots, e$, be a $C^{1}$ family of orthonormal normal vectors to $f\left(M^{d}\right)$ at $f(u)$, so that, in terms of Euclidean scalar products $N^{\lambda} \cdot N^{\kappa}=\delta^{\lambda \kappa}$ and $f_{i} \cdot N^{\lambda}=0$ for all $i, \lambda$, where $f_{i}=\partial f / \partial u^{i}$. Thus we have the Gauss and Weingarten derivation formulae,

$$
\begin{aligned}
f_{i j} & =\Gamma_{i j}^{k} f_{k}+h_{i j}^{\lambda} N^{\lambda}, & h_{i j}^{\lambda} & =f_{i j} \cdot N^{\lambda}=-f_{i} \cdot N_{j}^{\lambda}, \\
N_{i}^{\lambda} & =-h_{i j}^{\lambda} g^{j m} f_{m}+b_{i}^{\lambda \kappa} N^{\kappa}, & b_{i}^{\lambda \kappa} & =N_{i}^{\lambda} \cdot N^{\kappa}=-b_{i}^{\kappa \lambda} .
\end{aligned}
$$

Let $u=u(s), 0 \leqq s \leqq c$, be an arclength parametrization of a subarc of $L^{1}, u^{\prime}=d u / d s$, and $f^{\prime}=d f(u(s)) / d s=f_{i} u^{i^{\prime}}$. Thus (1) is equivalent to

$$
h_{i j}^{\lambda}(u(s)) u^{i^{\prime}}(s)=0 \text { for all } \lambda \text { and } 0 \leqq s \leqq c .
$$

The normal spaces (or tangent spaces) of $f\left(M^{d}\right)$ at $f(u(s))$ are independent of $s$ if and only if $C \cdot N^{\lambda}(u(s)) \equiv 0$ for all $\lambda, s$ and all vectors $C \in E^{d+e}$ tangent to $f\left(M^{d}\right)$ at $f(u(0))$. Put

$$
y^{\lambda}=C \cdot N^{\lambda}(u(s)), \text { so that } y^{\lambda}=0 \text { at } s=0 .
$$

From $y^{\lambda^{\prime}}=C \cdot N_{i}^{\lambda} u^{i^{\prime}}$ and (4.2),

$$
y^{\lambda^{\prime}}=-h_{i j}^{\lambda} u^{i^{\prime}} g^{j m}\left(C \cdot f_{m}\right)+b_{i}^{\lambda \kappa} u^{i^{\prime}} y^{\kappa} .
$$

Thus, (4.3) implies that

$$
y^{\lambda^{\prime}}=b_{i}^{\lambda \kappa} u^{i^{\prime}} y^{\kappa} \quad \text { and } \quad y(0)=0
$$

and hence that $y^{\kappa}(s) \equiv 0$ for any vector $C$ tangent to $f\left(M^{d}\right)$ at $f(u(0))$. Consequently, (1) implies (2). Conversely, let (2) hold, so that $y^{\lambda}(s) \equiv 0$ for any vector $C$ in the tangent space to $f\left(M^{d}\right)$ at $f(u(t)), 0 \leqq t \leqq c$. Then (4.4) gives

$$
0=y^{\lambda^{\prime}}=-h_{i j}^{\lambda} u^{i^{\prime}} g^{j m}\left(C \cdot f_{m}\right)
$$

Choosing $C=f_{k}(u(t))$ gives (4.3). Thus (1) and (2) are equivalent.

Suppose now that $M^{d}$ has nonnegative sectional curvatures and that $L^{1}$ is a geodesic arc. Then, by (4.1) and the fact that $u(s)$ is a geodesic,

$$
f^{\prime \prime}=h_{i j}^{\lambda} N^{\lambda} u^{i^{\prime}} u^{j^{\prime}} \text {. }
$$


The nonnegativity of the sectional curvatures of $M^{d}$ are expressed by the inequality

$$
\left(h_{i j}^{\lambda} \xi^{i} \xi^{j}\right)\left(h_{m n}^{\lambda} \eta^{m} \eta^{n}\right)-\left(h_{i j}^{\lambda} \xi^{i} \eta^{j}\right)\left(h_{m n}^{\lambda} \xi^{m} \eta^{n}\right) \geqq 0
$$

for all $\xi=\left(\xi^{1}, \ldots, \xi^{d}\right), \eta=\left(\eta^{1}, \ldots, \eta^{d}\right)$.

Since $f: M^{d} \rightarrow E^{d+e}$ is an isometric immersion, $s$ is also arclength on the arc $f(u(s)), 0 \leqq s \leqq c$. Thus $L_{1}=f\left(L^{1}\right)$ is line segment if and only if $f^{\prime \prime}=0$ or, equivalently,

$$
h_{i j}^{\lambda}(u(s)) u^{i^{\prime}} u^{j^{\prime}}=0 \text { for } \lambda=1, \ldots, e \text { and } 0 \leqq s \leqq c .
$$

If $\xi=u^{\prime}$ in (4.6), it follows that (4.7) holds if and only if $h_{i j}^{\lambda} u^{i^{\prime}} \eta^{j}=0$ for all $\eta$ and $\lambda$; i.e., (3). This proves the equivalence of (1) and (3).

The next proposition concerns the parenthetical part of Corollary 1.2 and is an obvious consequence of Toponogov's factorization theorem [15] (cf. ( $\left.T_{k j}\right)$ in $\S 5)$ on the product structure of complete manifolds of nonnegative sectional curvature containing "lines".

Proposition 4.2. Let $M^{d}$ be a complete Riemann manifold of class $C^{3}$ (or of class $C^{2}$ having a continuous curvature tensor as, e.g., in [5, p. 283]) containing a "line" $L^{1}$ and having nonnegative sectional curvatures. Then a tangent vector in $T_{p}\left(L^{1}\right)$ for $p \in L^{1}$ is a nullity vector at $p$. In particular, if through one (hence every) point $p \in M^{d}$, there passes $\mu_{0}$ linearly independent "lines", then the nullity satisfies $\mu(p) \geqq \mu_{0}$ for all $p \in M^{d}$.

The next proposition is unrelated to this paper, but is an "intrinsic analogue" of the last part of Proposition 4.1.

Proposition 4.3. Let $M^{d}$ be a Riemann manifold of class $C^{3}$ (or of class $C^{2}$ having a continuous curvature tensor as, e.g., in [5, p. 283]). Let $p \in M^{d}$ and suppose that either $K_{p}(\Sigma) \geqq 0$ or $K_{p}(\Sigma) \leqq 0$ holds for the curvatures $K_{p}(\Sigma)$ at $p$ of all 2-sections $\Sigma$ through $p$. Let $z \in T_{p}\left(M^{d}\right)$. Then $z$ is a nullity vector if and only if $K_{p}(\Sigma)=0$ holds for all 2-sections $\Sigma$ through $p$ with $z \in T_{p}(\Sigma)$.

Proof. The definition of a nullity vector trivially implies that $K_{p}(\Sigma)=0$ for all 2-sections through $p$ with $z \in T_{p}(\Sigma)$.

Conversely, suppose that $K_{p}(\Sigma)$ for all such 2-sections. In appropriate local coordinates, with $z=\left(z^{1}, \ldots, z^{d}\right)$,

$$
R_{i j k m} x^{i} x^{k} z^{j} z^{m}=0 \text { for all } x=\left(x^{1}, \ldots, x^{d}\right) .
$$

Since $K_{p}(\Sigma) \geqq 0$ or $K_{p}(\Sigma) \leqq 0$ for all 2-sections $\Sigma$ through $p$, the vector $z$ is a null vector for the symmetric matrix $\left(R_{i j k m} x^{i} x^{k}\right)=\left(R_{k m i j} x^{i} x^{k}\right)$, for $j, m=1, \ldots, d$,

$$
R_{i j k m} x^{i} x^{k} z^{m}=0 \text { for all } j \text { and } x \text {. }
$$

Thus, for fixed $j$, this form in $x$ is skew,

$$
\left(R_{i j k m}+R_{k j i m}\right) z^{m}=0 \text { for all } i, j, k .
$$


From the standard relation $R_{i j k m}+R_{k i j m}=R_{k j i m}$, we obtain

$$
\left(2 R_{i j k m}+R_{k i j m}\right) z^{m}=0 .
$$

By (4.8), $R_{k i j m} z^{m}=-R_{j i k m} z^{m}=R_{i j k m} z^{m}$, so that

$$
R_{i j k m} z^{m}=0 \text { for all } i, j, k \text {. }
$$

Hence $z$ is a nullity vector.

5. Differentiability assumptions in theorems of Rauch and Toponogov. The assumption $M^{d} \in C^{k}$ implies that, in local coordinates, the metric tensor is of class $C^{k-1}$. In general, $k=2$ does not permit the definition of sectional curvature on $M^{d}$. But the existence of an isometric immersion $f: M^{d} \rightarrow E^{d+e}$ of class $C^{2}$ implies that there exists a continuous curvature tensor expressible in terms of second fundamental forms; cf., e.g., [5, pp. 283-284]. Thus the statements in $\S 1$ are meaningful even for $k=2$.

When $M^{d} \in C^{k}, k \geqq 2$, has a continuous curvature tensor of class $C^{j}, 0 \leqq j \leqq k-2$ (for example, if there exists an isometric immersion $f: M^{d} \rightarrow E^{d+e}$ of class $C^{j+2}$ ), then there exist local geodesic coordinates of class $C^{j+1}$; cf. [5].

No further remarks are needed as to "differentiability" in the proofs of Corollary 1.1 and Lemma 1.1. The proofs of the other results of $\$ 1$ depend however on the factorization theorem of Toponogov [15], called $\left(\mathrm{T}_{\infty \infty}\right)$ below, which in turn is based on his comparison theorem [14], see $\left(\mathrm{CT}_{\infty}\right)$ below. Consider the validity of the following two assertions, $\left(\mathrm{T}_{k j}\right)$ and $\left(\mathrm{CT}_{k}\right)$ :

( $\mathrm{T}_{k j}$ ) Assume that (i) $M^{d} \in C^{k}$ is a complete Riemann manifold with nonnegative sectional curvatures and that $(\alpha)$ there exist $m$ linearly independent "lines" through some point $p \in M^{d}$. Then there exists a complete Riemann manifold $M^{d-m} \in C^{j}$ such that $M^{d}=E^{m} \times M^{d-m}$.

$\left(\mathrm{CT}_{k}\right)$ Assume that (i) holds. If $a b c$ is a triangle in $M^{d}$ with minimizing geodesics as sides and $A B C$ is a triangle in $E^{2}$ with corresponding sides equal to those of $a b c$, then corresponding angles of $A B C$ do not exceed those of $a b c$.

On the one hand, Toponogov's proof [15] of $\left(\mathrm{T}_{\infty}\right)$ makes it clear that if $\left(\mathrm{CT}_{k}\right)$ holds for $M^{d}$ and if there exist (local) geodesic coordinates of class $C^{j}$ on $M^{d}$ with $j \leqq k$, then $\left(\mathrm{T}_{k j}\right)$ holds. On the other hand, we shall point out below that the proof of Toponogov's comparison theorem in [4, pp. 182-194], can be modified to yield

(a) $\left(\mathrm{CT}_{2}\right)$ is valid, provided that $M^{d}$ has a continuous curvature tensor (for example, in the sense employed in [5, p. 283]).

This gives, therefore, the following:

(b) $\left(\mathrm{T}_{k, k-1}\right)$ is valid for $k \geqq 2$ provided that $M^{d} \in C^{k}$ has a curvature tensor of class $C^{k-2}$ (e.g., if there exists an isometric immersion $f: M^{d} \rightarrow E^{d+e}$ of class $C^{k}$ ). In particular, $\left(\mathrm{T}_{k j}\right)$ is valid for $k \geqq 3,1 \leqq j \leqq k-2$.

These remarks indicate the correctness of the proofs above under the stated differentiability conditions. It is clear that one can obtain assertions analogous to 
those of $\S 1$ if one replaces the assumptions " $M^{d} \in C^{k}$ and $f \in C^{k}, k \geqq 2$ " by " $M^{d} \in C^{k}, k \geqq 3$, and $f \in C^{j}, 1 \leqq j \leqq k-2$ " and the corresponding part of the conclusion by " $M^{d-m} \in C^{k-2}$ and $g \in C^{j}$ ".

The remainder of this section is devoted to a verification of (a). The proof of Toponogov's comparison theorem $\left(\mathrm{CT}_{\infty}\right)$ in [4] depends essentially on two results: Rauch's comparison theorem and a result of A. D. Alexandrov $(d=2)$ and Toponogov $(d \geqq 2)$. The latter is stated as Lemma 2 in [4, p. 187], and corresponds to $\left(\mathrm{b}_{0}\right)$ below with a $C^{\infty}$ assumption. Thus the proof of $\left(\mathrm{CT}_{\infty}\right)$ in [4] can be modified to yield $(a)$ if the following assertions $\left(a_{0}\right),\left(b_{0}\right)$ are verified. (For other proofs of $\left(\mathrm{CT}_{\infty}\right)$ or related results, see [1], [9].)

$\left(\mathrm{a}_{0}\right)$ Rauch's comparison theorem is valid for manifolds of class $C^{2}$ having continuous curvature tensors.

$\left(\mathrm{b}_{0}\right)$ Let $M^{d} \in C^{2}$ be a complete Riemann manifold; $p, q, r$ distinct points of $M^{d}$; $\beta, \gamma$ minimizing geodesics from $p$ to $q$ and $p$ to $r$, respectively; $r \notin \beta ; q_{1}, q_{2}, \ldots$, points of $\beta, p \neq q_{n} \rightarrow p$ as $n \rightarrow \infty ; \gamma_{n}$ a minimizing geodesic from $q_{n}$ to $r ; \theta$ the angle from $\gamma$ to $\beta$ at $p$; and $\theta_{n}$ the angle from $\gamma_{n}$ to $\beta$ at $q_{n}$. Then $\lim \sup \theta_{n} \leqq \theta$ as $n \rightarrow \infty$.

On $\left(\mathrm{a}_{0}\right)$. Let $M^{d}$ be a Riemann manifold of class $C^{2}$ having a continuous curvature tensor. Consider local coordinates $u=\left(u^{1}, \ldots, u^{d}\right)$ on a neighborhood of $M^{d}$. Let $u=u(s, t)$ be a 1-parameter family of geodesics having initial conditions $u(0, t), u^{\prime}(0, t)=[d u(s, t) / d s]_{s=0}$ of class $C^{1}$ for small $|t|$. Then, by [5, pp. 283-284], $u(s, t)$ and $u^{\prime}(s, t)$ are of class $C^{1}$ in $(s, t)$; furthermore, if $v_{j}=g_{j k} u^{k^{\prime}}$, then $\partial^{2} u^{i} \partial s \partial t$ $=u_{t}^{i^{\prime}}$ and $\left(v_{j t}-\Gamma_{j m}^{k} v_{k} u_{t}^{m}\right)^{\prime}$ exist, are continuous, and satisfy the system of differential equations

$$
\begin{gathered}
u_{t}^{i^{\prime}}+\Gamma_{j k}^{i} u_{t}^{j} u^{k^{\prime}}=g^{i j}\left(v_{j t}-\Gamma_{j m}^{k} v_{k} u_{t}\right), \\
\left(v_{j t}-\Gamma_{j m}^{k} v_{k} u_{t}^{m}\right)^{\prime}-\Gamma_{j k}^{i}\left(v_{i t}-\Gamma_{i n}^{m} v_{m} u_{t}^{n}\right) u^{k^{\prime}}=-g_{i n} R_{m j k}^{i} u^{n^{\prime}} u^{m^{\prime}} u_{k}^{t} .
\end{gathered}
$$

The left side of (5.1) is the $i$ th component of $D u_{t}$, the covariant derivative of the covariant vector $u_{t}=\partial u / \partial t$ along the geodesic $u=u(\cdot, t)$. The left side of (5.2) is the $j$ th component of the covariant derivative of a contravariant vector along the same geodesic for fixed $t$. Since the covariant derivative of the metric tensor is 0 , (5.1) and (5.2) show that, for a fixed $t, w=u_{t}=\partial u / \partial t$ satisfies the Jacobi equation in the standard form

$$
D(D w)+R\left(w, u^{\prime}\right) u^{\prime}=0
$$

(In particular, $D(D w)$ exists and is a continuous function of $(s, t)$.) The standard theory of existence, uniqueness, and disconjugacy for this type of normal, linear, second order, selfadjoint system (cf., e.g., [7, pp. 384-396]) and the usual proofs of Rauch's comparison theorem (cf., e.g., [4, pp. 178-181]) give $\left(\mathrm{a}_{0}\right)$.

On $\left(\mathrm{b}_{0}\right)$. The theorem $\left(\mathrm{b}_{0}\right)$, with $C^{2}$ replaced by $C^{\infty}$, is deduced in [4, pp. 187-189], from a result similar to 
Proposition 5.1. Let $M^{d} \in C^{2}$ be a complete Riemann manifold and $M_{0} \subset M^{d} a$ compact set. Then there exist constants $\varepsilon=\varepsilon\left(M_{0}\right)>0, C=C\left(M_{0}\right)$ with the property that, for $q \in M_{0} ; x, y \in T_{q}\left(M_{0}\right) ;$ and $\|x\|,\|y\| \leqq \varepsilon$,

$$
\left|\operatorname{dist}\left(\exp _{q} x, \exp _{q} y\right)-\|x-y\|\right| \leqq C\left(\|x\|^{2}+\|y\|^{2}\right) .
$$

In (5.4), $\exp _{q} x=u(1)$, where $u(s)=\exp _{q} s x$ is a (not necessarily unique) geodesic satisfying $u(0)=q, u^{\prime}(0)=x$.

The proof of $\left(\mathrm{b}_{0}\right)$ in [4] uses a form of Proposition 5.1 in which $C\left(\|x\|^{2}+\|y\|^{2}\right)$ in (5.4) is replaced by $o\left(\left\|x_{n}\right\|^{2}+\left\|y_{n}\right\|^{2}\right)$ if $q=q_{n} \rightarrow p$ in $M^{d}$ and $x=x_{n}, y=y_{n}$ $\rightarrow 0 \in T_{p}\left(M^{d}\right)$ in $T\left(M^{d}\right)$. Actually, the proof of $\left(b_{0}\right)$ in [4] is valid if " $o$ " is weakened to " $O$ ".

Proof of Proposition 5.1. Let $p \in M^{d} ; u=\left(u^{1}, \ldots, u^{d}\right)$ local coordinates on a neighborhood $N$ of $p: u=0 ; G^{2}(u)=\left(g_{i j}(u)\right)$ the first fundamental matrix; and $G(u)$ the positive definite, symmetric square root of $G^{2}(u)$. Below, we shall identify a point of $N$ and its local coordinates. $N$ will denote a suitable neighborhood of $p$ and $C_{1}=C_{1}(N)$ a constant depending only on $N ; N$ and $C_{1}$ are not always the same.

Let dist $(\cdot, \cdot)$ be the metric on $M^{d} ;|\xi|$ the Euclidean length of $\xi \in R^{d}$; and $\|\xi\|_{u}=|G(u) \xi|$ the norm of $\xi \in T_{u}\left(M^{d}\right)$. For a suitable choice of $N$, there is a constant $C_{1}=C_{1}(N)$ such that

$$
\begin{gathered}
|\xi| / C_{1} \leqq|G(u) \xi| \leqq C_{1}|\xi| \\
|[G(u)-G(v)] \xi| \leqq C_{1}|u-v| \cdot|\xi|
\end{gathered}
$$

for all $\xi \in R^{d}$ and $u, v \in N$. There also exists a constant $\varepsilon_{1}=\varepsilon_{1}(N)>0$ such that if $u=u(s), 0 \leqq s \leqq c$, is an arclength parametrization of a geodesic of length $c \leqq \varepsilon_{1}$ with $u(0) \in N$, then, by the differential equations for geodesics, $\left|u^{\prime \prime}\right| \leqq C_{1}$ and

$$
\begin{gathered}
\left|\int_{0}^{c}\right| G(u(0)) u^{\prime}(s)|d s-c| \leqq C_{1} c^{2}, \\
\left|u(c)-u(0)-c u^{\prime}(s)\right| \leqq C_{1} c^{2} \text { for } 0 \leqq s \leqq c .
\end{gathered}
$$

Since

$$
|c| G(u(0)) u^{\prime}(s)|-| G(u(0))[u(c)-u(0)]|| \leqq C_{1} c^{2}
$$

we have

$$
|c-| G(u(0))[u(c)-u(0)]|| \leqq C_{1} c^{2} .
$$

In particular, $c / C_{1}-C_{1} c^{2} \leqq|u(c)-u(0)| \leqq C_{1} c+C_{1} c^{2}$; so that if $0<c \leqq \varepsilon_{1}, \varepsilon_{1}=\varepsilon_{1}(N)$ is sufficiently small, and $u(0) \in N$, then

$$
c / C_{1} \leqq|u(c)-u(0)| \leqq C_{1} c .
$$


Let $q \in N, 0 \neq x \in T_{q}\left(M^{d}\right)$, and $u(s)=\exp _{q}\left(s x /\|x\|_{q}\right)$ for $0 \leqq s \leqq\|x\|_{q} \leqq \varepsilon_{1}$. Then (5.8) gives

$$
\left|\exp _{q} x-q-x\right| \leqq C_{1}\|x\|_{q}^{2} \text {. }
$$

This inequality is trivial if $x=0$. From this inequality and a corresponding one for $y, y \in T_{q}\left(M^{d}\right)$ and $\|y\|_{q} \leqq \varepsilon_{1}$,

$$
\left|\left(\exp _{q} x-\exp _{q} y\right)-(x-y)\right| \leqq C_{1}\left(\|x\|_{q}^{2}+\|y\|_{q}^{2}\right) .
$$

Let $u=u(s), 0 \leqq s \leqq c=\operatorname{dist}\left(\exp _{q} x, \exp _{q} y\right)$ be a minimizing geodesic joining $u(0)$ $=\exp _{q} x$ and $u(c)=\exp _{q} y$. Then, by (5.9), (5.10) and (5.11),

$$
\left|\operatorname{dist}\left(\exp _{q} x, \exp _{q} y\right)-\right| G(u(0))\left[\exp _{q} x-\exp _{q} y\right]|| \leqq C_{1}\left(\|x\|_{q}^{2}+\|y\|_{q}^{2}\right) .
$$

The analogue of (5.10) applied to the geodesic $\exp _{q}\left(s x /\|x\|_{q}\right), 0 \leqq s \leqq\|x\|_{q}$, gives

$$
\|x\|_{q} / C_{1} \leqq\left|\exp _{q} x-q\right| \leqq C_{1}\|x\|_{q},
$$

and (5.6) applied to $u(0)=\exp _{q} x, v=q$ gives

$$
|[G(u(0))-G(q)] \xi| \leqq C_{1}\|x\|_{q}|\xi| \quad \text { for all } \xi \in R^{d} .
$$

Thus, (5.11), (5.12) and $\|x-y\|_{q}=|G(q)(x-y)|$ imply that

$$
\mid \text { dist }\left(\exp _{q} x, \exp _{q} y\right)-\|x-y\|_{q} \mid \leqq C_{1}\left(\|x\|_{q}^{2}+\|y\|_{q}^{2}\right)
$$

for $q \in N$ and $x, y \in T_{q}\left(M^{d}\right),\|x\|_{q} \leqq \varepsilon_{1},\|y\|_{q} \leqq \varepsilon_{1}$. Proposition 5.1 now follows from the compactness of $M_{0}$.

\section{REFERENCES}

1. M. Berger, An extension of Rauch's metric comparison theorem and some applications, Illinois J. Math. 6 (1962), 700-712. MR 26 \#719.

2. E. Calabi and P. Hartman, The smoothness of isometries, Duke Math. J. (to appear).

3. S. S. Chern and N. H. Kuiper, Some theorems on the isometric imbedding of compact Riemann manifolds in Euclidean space, Ann. of Math. (2) 56 (1952), 422-430. MR 14, 408.

4. D. Gromoll, W. Klingenberg and W. Meyer, Riemannsche Geometrie im Grossen, Lecture Notes in Mathematics, no. 55, Springer-Verlag, Berlin, 1968. MR 37 \#4751.

5. P. Hartman, On exterior derivatives and solutions of ordinary differential equations, Trans. Amer. Math. Soc. 91 (1959), 277-293. MR 22 \#123.

6. - On isometric immersions in Euclidean space of manifolds with non-negative sectional curvatures, Trans. Amer. Math, Soc. 115 (1965), 94-109. MR 34 \#1968.

7. — Ordinary differential equations, Wiley, New York, 1964. MR 30 \#1270.

8. P. Hartman and L. Nirenberg, On spherical image maps whose Jacobians do not change sign, Amer. J. Math. 81 (1959), 901-920. MR 23A \#4106.

9. R. Kulkarni, Curvature and metric, Thesis, Harvard University, Cambridge, Mass., 1967.

10. B. O'Neill, Isometric immersion of flat Riemannian manifolds in Euclidean space, Michigan Math. J. 9 (1962), 199-205. MR 27 \#2941.

11. H. E. Rauch, A contribution to differential geometry in the large, Ann. of Math. (2) 54 (1951), 38-55. MR 13, 159.

12. R. Sacksteder, On hypersurfaces with no negative sectional curvatures, Amer. J. Math. 82 (1960), 609-630. MR 22 \#7087. 
13. E. Stiel, Isometric immersions of manifolds of nonnegative constant sectional curvature, Pacific J. Math. 15 (1965), 1415-1419. MR 32 \#6375.

14. V. A. Toponogov, A property of convexity of Riemannian manifolds of positive curvature, Dokl. Akad. Nauk SSSR 115 (1957), 674-676; English transl., Amer. Math. Soc. Transl. (2) 37 (1964), 283-285. MR 19, 979.

15. - - Riemannian spaces which contain straight lines, Dokl. Akad. Nauk SSSR 127 (1959), 977-979; English transl., Amer. Math. Soc. Transl. (2) 37 (1964), 287-290. MR 21 $\# 7520$.

THE JOHNS HOPKINS UNIVERSITY,

Baltimore, Maryland

UNIVERSITY OF WARWICK,

WARWICK, ENGLAND 